\title{
Temperature dependence of synchronized beating of cultured neonatal rat heart-cell networks with increasing age measured by multi-electrode arrays
}

\author{
Tsutomu Uchida ${ }^{1 *}$, Ryuichi Kitora ${ }^{1,2}$ and Kazutoshi Gohara ${ }^{1}$ \\ ${ }^{1}$ Division of Applied Physics, Faculty of Engineering, Hokkaido University, Sapporo, Hokkaido, Japan \\ ${ }^{2}$ Mitsubishi Electric Corporation, Hyogo, Japan (Current affiliation)
}

\begin{abstract}
Primary cultured cardiomyocytes are attracting interest as a screening base for new drugs. One promising approach for a steady supply of cells is to preserve cardiomyocytes at temperatures below physiological conditions. The aim of this study was to observe the change in activity of cultured heart-cell networks at temperatures lower than physiological conditions to investigate their appropriate preservation conditions. At various temperatures between $38^{\circ} \mathrm{C}$ and $22^{\circ} \mathrm{C}$, we measured the synchronized beating properties of neonatal rat ventricle heart-cells cultured on multi-electrode arrays. Synchronized beating rates decreased with temperature, but this temperature dependence varied according to the length of the culture period. For the heart-cell networks in the earlier stages of culture (1-3 days in culture), the beating rate decreased as the inter-beat interval increased, maintaining a constant rhythm. The propagation velocity of the synchronized field potential was approximately $5.9 \mathrm{~cm} / \mathrm{s}$ at physiological temperature and decreased weakly with temperature. Conversely, in the later stages of culture (approximately 1 week) the beating rate decreased with temperature as the variability in the inter-beat interval increased, especially below $30^{\circ} \mathrm{C}$. The propagation velocity of the synchronized potential signal was approximately $18 \mathrm{~cm} / \mathrm{s}$ at physiological temperature, which is three times larger than that in early stage. Then the propagation velocity drastically decreased with temperature down to similar range with that in early stage Therefore, we considered that the maturation of a heart-cell network stabilized the synchronized beating rhythm and increased its propagation velocity at physiological conditions, but simultaneously increased the temperature sensitivity of the beating properties of the network. These temperature dependences on the signal-propagation velocity is considered as the functional change of gap junctions during culture. Thus, a mature heart-cell network would be more difficult to preserve at lower temperatures.
\end{abstract}

\section{Introduction}

The demand for cardiomyocytes maintained in culture has recently increased. For example, freshly prepared cardiomyocytes are used for in vitro assays that assess the potential of new drugs [1]. Layered cardiomyocyte sheets reconstructed from isolated cells are also attracting considerable attention as three-dimensional biodegradable scaffolds for the transplantation of tissue-engineered heart grafts [2].

The supplementation of these cells with primary cardiomyocytes has been insufficient to meet the high industrial demand, as there has been little success in the cryopreservation and recovery [3]. Since donor heart is exposed to severe hypothermia and ischemia in the process of cardiac preservation and storage, it is vital that temperature and duration are controlled in order to maintain cardiac function [4-6]. In general, a body temperature less than $20^{\circ} \mathrm{C}$ causes cardiac arrest in an adult rat, often with irreversible tissue damage leading to death [7]. Hibernation studies have revealed that severe damage to mammalian hearts under hypothermia results mainly from the calcium-ion regulation mechanism [8]. These properties were also observed qualitatively in cultured cardiomyocytes, but the critical conditions were not consistent $[9,10]$.

Studies using in vitro assays of cardiomyocytes have revealed that the freshly harvested and cultured neonatal cardiomyocytes contract spontaneously and cyclically [11]. The growth of cardiomyocytes on a plate can be examined by observing the establishment of a stable synchronized-beating rhythm [12-18]. In the study of the synchronous behavior that occurs when isolated myocytes are assembled into a confluent sheet, microelectrode arrays (MEAs) are useful tools because they allow us to measure the electrophysiological maturation of cardiomyocytes over a wider spatiotemporal range with minimal disturbance [19-22]. The characteristics of the field potential recorded by MEAs have been associated with the intrinsic action potential of cardiomyocytes [23], which has been verified, at least for the initial depolarization by a sodium-ion current and for the subsequent repolarization by a potassium-ion current.

In the present study, we investigated the sensitivity of neonatal cardiomyocyte activities to temperature depending on the culture period (i.e., the level of maturation) of the heart-cell network. Toward this end, we compared the cell activities of these heart cells at a late stage of culture ( 6 to 8 days in vitro (DIV)) as a fully matured culture, and at an early stage of culture ( 1 to 3 DIV) as an immature culture. These activities are measured with a MEA at temperatures ranging from normothermia $\left(37^{\circ} \mathrm{C}\right)$ to severe hypothermia $\left(22^{\circ} \mathrm{C}\right)$. We then confirmed that the synchronized beating rates depressed with decreasing temperature in a different manner that depended on the number of days in culture. The decrease in the synchronized beating rate with

${ }^{\star}$ Correspondence to: Tsutomu Uchida, Division of Applied Physics, Faculty of Engineering, Hokkaido University, N13 W8 Kita-ku, Sapporo 060-8628, Japan, Tel: 81117066635; Fax: 81117066635; E-mail: t-uchida@eng.hokudai.ac.jp

Received: May 17, 2018; Accepted: June 05, 2018; Published: : June 08, 2018 

arrays

increasing variability of the inter-beat interval and the decrease in the propagation velocity of a field potential signal during the temperature reduction were more sensitive in the late-stage samples than in the early-stage samples. These qualitative observations of the synchronized beating and the temperature dependence of the propagation velocity of the beating suggest that the signal propagation mechanism is mainly controlled at the gap junction, and the provide a possible reason for the hypothermic intolerance in samples at different maturation stages.

\section{Materials and methods}

We only performed cell cultures in vitro in the present study. Animal works were not performed, so the approval from the Animal Care and Use Committee of our Institution was not required. We cultured the primary rat ventricle heart-cells dissociated from cut pieces of ventricle tissues (1-2 $\left.\mathrm{mm}^{3}\right)$ of neonatal Sprague-Dawley (SD) rats within 4 days of birth (Cosmo Bio Co., Ltd., Japan) as described previously [3,24]. Here we briefly show the preparation process of heart cells cultured on a MEA. The dissociated cells at about $2 \times 10^{5}$ cells $/ \mathrm{mL}$, which include more than $80 \%$ of cardiomyocytes [1], were suspended in a culture medium: Dulbecco's modified Eagle's medium containing $10 \%$ fetal calf serum, $100 \mathrm{U} / \mathrm{mL}$ penicillin, and $100 \mu \mathrm{g} / \mathrm{mL}$ streptomycin), and were kept at $4^{\circ} \mathrm{C}$ for $6 \mathrm{~h}$ during its transport. This process has been confirmed to not damage cardiomyocytes severely [24].

Approximately $1 \mathrm{~mL}$ of cell suspension was placed at the center of the MEA plate (MED probe, Alpha MED Scientific, Osaka, Japan) coated with $0.01 \%$ collagen (Cellgen IPC-15; Koken, Tokyo, Japan). This array consists of 64 planar microelectrodes arranged in an $8 \times 8$ grid embedded in the center of a transparent glass plate. The electrodes, which are $50-\mu \mathrm{m}$ square and coated with platinum black, were fabricated with an inter-electrode spacing of $450 \mu \mathrm{m}$, which allowed us to observe a wider area $\left(4 \times 4 \mathrm{~mm}^{2}\right)$ for the heart-cell network development. The cells were seeded to cover these electrodes by using a cloning ring with an inner diameter of $10 \mathrm{~mm}$, which resulted in a cell density of approximately $2.5 \times 10^{3}$ cells $/ \mathrm{mm}^{2}$.

The cells on the MED probe were cultured in an incubator (with a humidified atmosphere of $5 \% \mathrm{CO}_{2}$ and $95 \%$ air, at $37^{\circ} \mathrm{C}$ ). The cultured cells were observed with a phase-contrast microscope (Olympus, Tokyo, Japan; type CKX41) to check their shape, elongation, and beating state prior to and during the experiments. Usually the cultured heart cells began to beat spontaneously within $24 \mathrm{~h}$ initiating the culture and beat synchronously with neighboring cells within $48 \mathrm{~h}$ of their coming into contact with each other. Half of the cultured medium was changed every day during the cultivation. To observe the covering processes of the seeded cells over the plate surface, we also carried out the time-lapse observation [25] of the heart cells for several times. The detail processes of the time-lapse observations are described in the Supporting Information.

When we use the heart, cells cultured on the MED probe, the MED probe was mounted in a temperature-controlled box on the stage of the phase-contrast microscope. The morphology of cells and the beating properties of heart cells were continuously monitored and recorded using an S-VHS time-lapse video recorder (Victor, Tokyo, Japan; type SR-S990) and a CCD camera system (Olympus; type CS230B). The temperature of the apparatus was controlled by using a refrigerated circulator (Haake, Karlsruhe, Germany; type K-F3) with ethylene glycol solution in the temperature-controlled box. A thermocouple (type $\mathrm{T}$ ) positioned close to the specimen monitored the environmental temperature and showed that the culturing medium temperature was controlled within $\pm 0.1^{\circ} \mathrm{C}$. In order to avoid a temperature difference between the culturing medium and the environment, the temperaturechange rate was set sufficiently low, at approximately $0.05^{\circ} \mathrm{C} / \mathrm{min}$, for both the cooling and heating process. The same experiments were carried out more than four times $(n \geq 4)$ on the heart cells in different batches.

The spontaneous electrical activity of each heart-cell culture was recorded using an MED64 extracellular recording system (Alpha MED Scientific, Osaka, Japan) with a sampling rate of $20 \mathrm{kHz}$. A/D conversion was performed by using the MED64 conductor software (Alpha MED Scientific). Extracellular potential (field potential) traces usually contain biological and thermal noises of approximately $10-20 \mu \mathrm{V}$. In the present study, the beginning of the beating signal was determined to be when its amplitude exceeded a noise-based threshold. The coincidence of each beating and the field potential in the present study was confirmed by comparing the signal records with the video images. The beating rate was measured by counting the first large negative peaks during $1 \mathrm{~min}$. The inter-beat interval (IBI) was defined as the time difference between the starting time of a field-potential signal and that of the following one. Thus, IBI shows the periodicity of the beating independently from the beating rate measurement.

The data obtained in the present study is averaged at the indicated experimental conditions \pm the standard deviation (S.D.) of all measured data. Then, data are analyzed by one-way ANOVA with a Tukey-Kramer post-hoc test (Microsoft Excel 2010 and BellCurve for Excel) for at least 99\% confidence $(\mathrm{p}<0.05)$.

Immediately after the temperature-changing experiment on each setting day in culture, immunocytochemical arrays were performed on the culture on the MEA. The cultures were washed twice with phosphate-buffered saline (PBS; Invitrogen-Gibco, Carlsbad, CA, USA) and fixed with $4 \%$ formaldehyde in PBS for $10 \mathrm{~min}$, and then washed twice more with PBS at room temperature. The fixed cultures were permeabilized with $0.5 \%$ Triton X-100 (Sigma-Aldrich, St. Louis, MO) in PBS for 10 min and washed twice with PBS, then incubated for 30-40 min with PBS containing $0.5 \%$ bovine serum albumin (Wako, Tokyo, Japan), which we called BSA solution. The permeabilized cultures were incubated with primary antibodies in the BSA solution for $1 \mathrm{~h}$ and were rinsed three times with the BSA solution for $10 \mathrm{~min}$. The cultures were then incubated with secondary antibodies in the BSA solution for $1 \mathrm{~h}$ and were rinsed a further three times. Connexin-43 (Cx43) was detected by using anti-Cx43 rabbit IgG $(0.1 \%$, Sigma-Aldrich: product code C6291) as the primary antibody and $0.4 \%$ Alexa Fluor 488-labeled anti-rabbit IgG (Molecular Probes, Eugene, OR, USA) as the secondary antibody. a-Actinin was detected using anti-actinin mouse IgG (0.2\%, Sigma-Aldrich) as the primary antibody and $0.4 \%$ Alexa Fluor 546-labeled anti-mouse IgG (Molecular Probes) as the secondary antibody. To observe the cell nuclei, cultures were incubated with $2 \mu \mathrm{g} /$ mL Hoechst 33342 (Dojindo Laboratories, Kumamoto, Japan) for 10 min after incubation with secondary antibodies. Observations were performed using an epifluorescence microscope (Olympus, type IX-71) equipped with an Olympus $10 \times$ or $20 \times$ objective lens. The images were captured with a CCD camera system (Olympus, type DP71).

\section{Results}

\section{Microscopic observations of cultured ventricle heart-cells under normothermia conditions}

The dispersed rat heart cells adhered to and spread out on both the glass plate and electrodes of the MEA at the early stage of culture (within 1-3 day in vitro (DIV); Figure 1a). They started beating spontaneously at the rates of approximately 100 beats per min (bpm) 

arrays

within one day after plating. Then they gradually came into contact with neighboring cardiomyocytes, with which their beatings became synchronized, even though their original beating rates differed. The beating of most cardiomyocytes on MEAs becomes synchronized within 2 DIV under the conditions of this study (Figure S1; Video in the Supporting Information). The fluorescence images for $\alpha$-actinin (red), Cx43 (green) and nuclei (blue) in Figure 1b show that there were two or three isolated and elongated cardiomyocytes on an electrode $\left(50 \times 50 \mu \mathrm{m}^{2}\right)$. Some of them were connected with several neighboring cardiomyocytes mainly at the tip of the cell by forming a gap junction, which is shown by the expression of $\mathrm{Cx} 43$ (Figure 1b) at the early stage of culture.

During the middle stage (within 4-5 DIV; Figures 1c and 1d), cells gradually formed a cell domain (typically $\sim 10$ cells as shown in Figure 1d) that included several cardiomyocytes and non-cardiomyocytes and all cardiomyocytes in the cell domain beat synchronously. The heart cells changed in shape from an isolated form (observed at $1 \mathrm{DIV}$, figure not shown) to a closely packed form (Figures $1 \mathrm{c}$ and $1 \mathrm{~d}$ ). Cx43 staining was most pronounced along the cell-to-cell borders. Simultaneously, non-cardiomyocytes (mostly fibroblasts), recognized as a cell having nucleus (blue) but not $\alpha$-actinin (red) in Figure 1d, multiplied at the openings between cell domains such that they became confluent. The synchronized beating rate usually peaked (at approximately $140 \mathrm{bpm}$ under this study conditions) at 3-4 DIV, which almost coincided with the time at which the cell sheet became confluent. This is the same as reported previously [24].

At the late stage of culture (6-8 DIV), the MEA surface was completely covered with cells (Figures 1e and 1f), in which the neighboring cells had connected with forming larger cell domains, while expressing $\mathrm{Cx} 43$ at the cell-to-cell boundary (Figure 1f). The space between the cell domains existed in earlier stages was filled with non-cardiomyocyte cells, binding the domains and forming a synchronized cell sheet on the MEA. The synchronized beating rate was usually slower (approximately $30 \mathrm{bpm}$ ) in the late stage of culture than in earlier stages (Figure S3; Video in the Supporting Information), which coincided with the results reported previously [24].

The time-lapse observation of the seeded heart cells qualitatively confirmed the validity of the morphologic observations shown in Figure 1. The immunofluorescence observations showed that most of heart cells at the late stage were non-cardiomyocyte cells (approximately two-thirds in number), so we estimated the initial cell ratio between cardiomyocyte and non-cardiomyocyte by the reverse observation of the time-lapse video. Then we estimated that the ratio of cardiomyocytes in the seeded cells was $0.78 \pm 0.02(n=5)$. This is consistent with the previous work [1].

This reverse-observation technique also showed that several cardiomyocytes divided once after plating (Figure S5 in the Supporting Information). Although it has been considered that cardiomyocytes do not divide after differentiation, we found that about $9 \%$ of cardiomyocytes were divided after plating. Therefore, cardiomyocytes come to be in the cell domain at the middle stage not only by migration and by connection with neighboring cardiomyocytes, but also as a result of division even partly.

\section{Temperature dependence of beating rate and inter-beat interval in different stage of culture as measured using an MEA}

When the temperature of the cell environment was gradually decreased from $38^{\circ} \mathrm{C}$ to $22^{\circ} \mathrm{C}$ at a rate of $-0.05^{\circ} \mathrm{C} / \mathrm{min}$, the beating rate of the cell sheets gradually decreased. Figure 2 shows the temperature dependence of the average beating rate in the early stage (open circles: $2 \mathrm{DIV}, n=4$ ), in the middle stage (solid squares: $4 \mathrm{DIV}, n=4$ ), and in the late stage of culture (solid circles: $7 \mathrm{DIV}, n=6$ ). The synchronized beating rate for the early stage sample decreased almost linearly (approximately $-5.3 \mathrm{bpm} /{ }^{\circ} \mathrm{C}$ ) across the whole temperature range studied, except that for the late stage samples, in which the inflexion point occurred at approximately $30^{\circ} \mathrm{C}$; the change in the synchronized beating rate
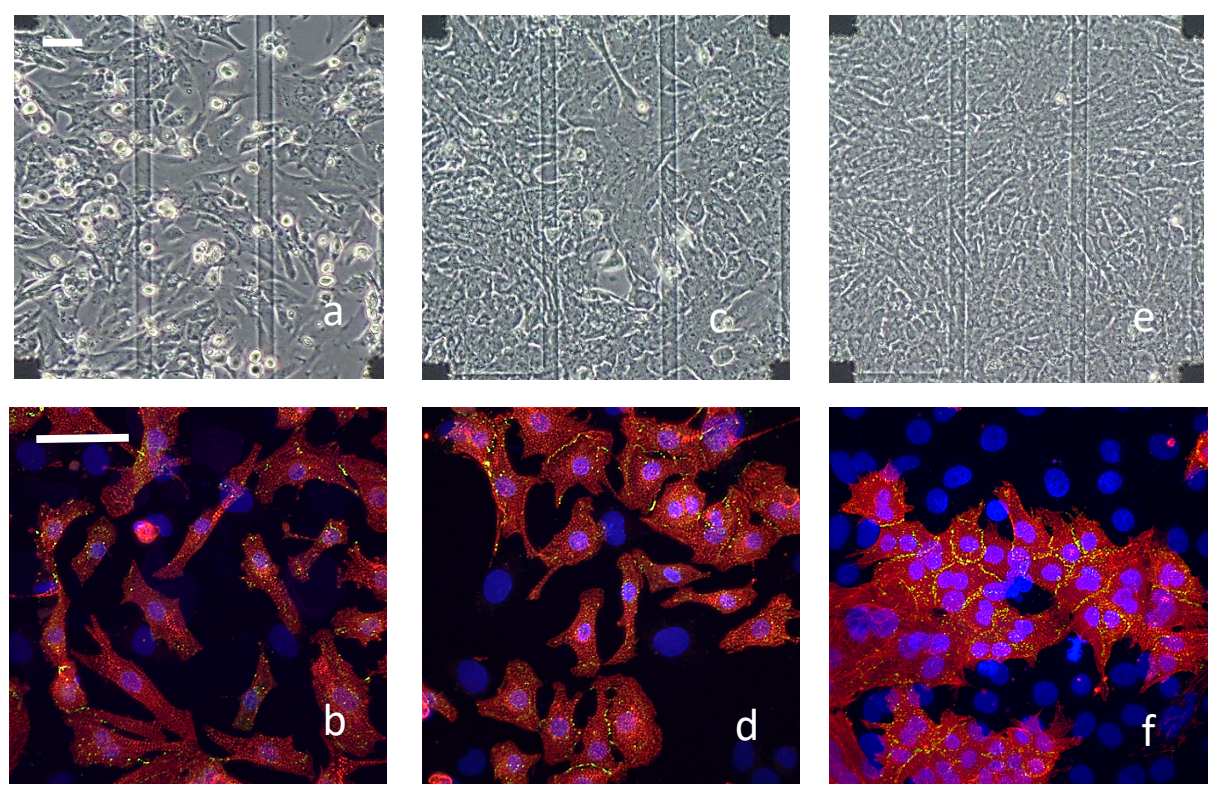

Figure 1. Microscopic and immunofluorescence staining images of different cultured heart cells. Microscopic images (upper) and immunofluorescence staining images (lower) in the early stage (a and b: 2 DIV), in the middle stage (c and d: 4 DIV) and in the late stage (e and f: 7 DIV). The block squares observed at the corners of the microscopic images are the electrode arrays that were located $450 \mu \mathrm{m}$ apart (scale bar: $50 \mu \mathrm{m}$ ). Transparent lines are electric wires made of ITO. Immunofluorescence staining images were constructed by merging three staining images (red: $\alpha$-actinin, green: Cx43, blue: nuclei) with a higher magnification (scale bar: $50 \mu \mathrm{m}$ ). A cardiomyocyte is identified by the expression of $\alpha$-actinin with a nucleus. Only the nuclei of non-cardiomyocytes are stained. $\mathrm{Cx} 43 \mathrm{~s}$ were mostly expressed by cardiomyocytes and was distributed mainly at the cell-to-cell boundaries 


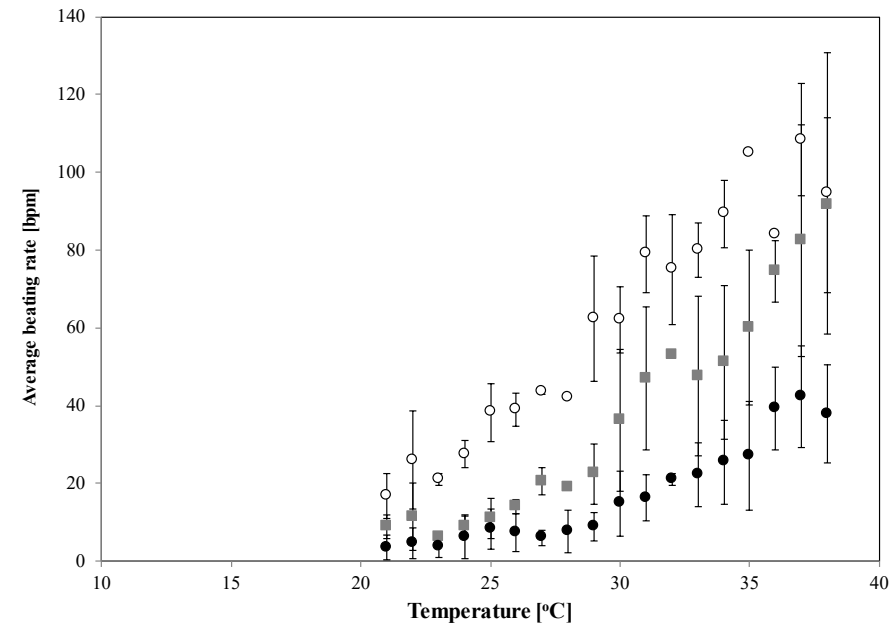

Figure 2. Temperature dependence of synchronized beating rates. Mean beating rates in the early stage (open circles; 2 DIV, $n=4$ ), middle stage (solid squares; 4 DIV, $n$ 4) and late stage of culture (solid circles; 7 DIV, $n=6$ ), measured at each temperature. Each erro bar shows S.D. measured 10 times in each sample and repeated for $n$ times. Statistical analysis indicates that there are differences between each culture stage $(*: p<0.05$, by a Tukey-Kramer test)

was greater at higher temperatures (approximately $-3.1 \mathrm{bpm} /{ }^{\circ} \mathrm{C}$ ) than at lower temperatures (approximately $-1.1 \mathrm{bpm} /{ }^{\circ} \mathrm{C}$ ). For the middle stage samples, the temperature dependence of the beating rates was intermediate between that of the early and late stage. Statistical analysis indicates that there are differences on the temperature dependences of the beating rate between three culture stages $(\mathrm{p}=0.01,0.04$ and $<0.001$ for early-middle stages, for middle-late stages, and early-late stages, respectively, by a Tukey-Kramer test). This temperature dependence was found to be reversible when the temperature increased from $22^{\circ} \mathrm{C}$ to $38^{\circ} \mathrm{C}$ at a rate of $+0.05^{\circ} \mathrm{C} / \mathrm{min}$. We did not observe any significant hysteresis in the beating rate within the measurement uncertainty.

To examine the periodicity of synchronized beating, we estimated the temperature dependence of the inter-beat interval (IBI) for both the early and late stage samples. The early stage samples showed that the IBI gradually increased from approximately $0.6 \mathrm{~s}$ at $38^{\circ} \mathrm{C}$ to approximately $4 \mathrm{~s}$ at $22^{\circ} \mathrm{C}$ with smaller fluctuations at each temperature (Figure $3 \mathrm{a}$ ). Then we hypothesized that the synchronized beating was maintained periodically with a gradual increase in IBI at lower temperatures in the early stage cultures. This is the main reason of synchronized beating-rate decreasing with temperature. Conversely, the late stage samples exhibited a different dependence on temperature. As shown in Figure $3 \mathrm{~b}$, the IBI gradually increased from approximately $1.5 \mathrm{~s}$ at $38^{\circ} \mathrm{C}$ to $3 \mathrm{~s}$ at $32^{\circ} \mathrm{C}$, and to $6 \mathrm{~s}$ at $30^{\circ} \mathrm{C}$, with small fluctuations in this temperature range. However, fluctuations in the IBI became greater when the temperature dropped below $30^{\circ} \mathrm{C}$. This critical temperature coincides with the inflexion in the relationship between temperature and the synchronized beating rate that is shown in Figure 2. Even though IBIs fluctuate greatly at lower temperatures, the microscopic observations confirmed that the heart-cell network in the late stage exhibited synchronized beating (Figure S4; Video in the Supporting Information).

We compared the profiles of the action potentials measured at the different temperatures to determine how the activity of the cardiomyocytes changed with temperature. Figure $4 \mathrm{a}$ shows the typical field potential profile measured on one electrode in the late stage culture at three temperatures: 38,30 , and $22^{\circ} \mathrm{C}$. This figure indicates that when the temperature decreased the profile of the initial negative peaks (denoted as the fast $\mathrm{Na}^{+}$current [22]) changed smaller and slower. An example of the temperature dependence of the amplitude of the negative peak is provided in Figure S7 in the Supporting Information. It is also notable that the subsequent small positive peaks (denoted as $\mathrm{K}^{+}$ current [22]) occurred later and became smaller at lower temperatures. These field potential profiles are consistent with the contraction of cardiomyocytes observed under the microscope. Thus we conclude that the typical beating of cardiomyocytes in the late culture stage became weaker and slower at lower temperatures. This is qualitatively consistent with a previously reported study [26]

Conversely, Figure 4c shows the field potential profiles of early stage cultures at different temperatures. Although the initial negative peak became smaller slightly with temperature, the amplitude decrease of the first negative peak with temperature was small compared to that of the late stage cultures (Figure 4a). Since the subsequent positive peak is very small in the early stage culture, we cannot confirm that the time period of one stroke of beating had changed. Therefore, it is considered that the beating activity of cardiomyocytes at the early stage were less sensitive to temperature. The microscopic observations support this relationship between temperature and beating activity (Figure S2; Video in the Supporting Information).

The field potentials in the middle stage culture were intermediate between those of the early and late stage; the $\mathrm{Na}^{+}$current was relatively insensitive to temperature, whereas the $\mathrm{K}^{+}$current responded later at lower temperatures (Figure $4 \mathrm{~b}$ ).

\section{Temperature dependence of the propagation velocity at different stages of culture}

Since we observed that the cellular connections were maintained at all temperatures investigated in the present study, we measured the propagation velocity of the field potential signal at three temperatures $22^{\circ} \mathrm{C}, 30^{\circ} \mathrm{C}$, and $38^{\circ} \mathrm{C}$ for both early and late stage cultures. We defined the starting point of a spontaneous synchronized beating as the electrode that recorded the earliest time of the minimal field potential of all the electrodes. Then the time interval between the start of beating and this earliest time was calculated as an offset time for each electrode.

Figure 5a (upper column) shows the typical map of the offset time interval between the minimal field potential at each electrode for the late stage cultures. At $38^{\circ} \mathrm{C}$, the dashed circle indicates the starting electrode of the showing beat. The starting point was usually located with a single fixed electrode in more than $90 \%$ of cases at this temperature. The lower column shows the offset-time interval variation with the distance between the electrodes. This diagram shows that the beating signal propagates almost concentrically from the starting point to all active electrodes because the offset time depends almost linearly with the distance. Therefore, it is possible to estimate the propagation velocity by the inclination of the regression line.

In order to obtain the average propagation velocity of the synchronized field potential, we measured 10 synchronized signals at each temperature (in the time period from 5 to $15 \mathrm{~min}$ ) for three different cultures. Then we obtained the mean propagation velocity $\left(v_{L}\right)$ of approximately $18 \pm 4.4 \mathrm{~cm} / \mathrm{s}$ for this condition. The variation in this value resulted mainly from the variation between the three different cultures because the variation in propagation velocity among the 10 synchronized beatings from the same culture was smaller. The mean propagation velocity obtained was similar to those reported previously $(14-35 \mathrm{~cm} / \mathrm{s})$ [20]. Conversely, when the temperature decreased to $30^{\circ} \mathrm{C}$ and to $22^{\circ} \mathrm{C}$, the starting point of the synchronized 

arrays
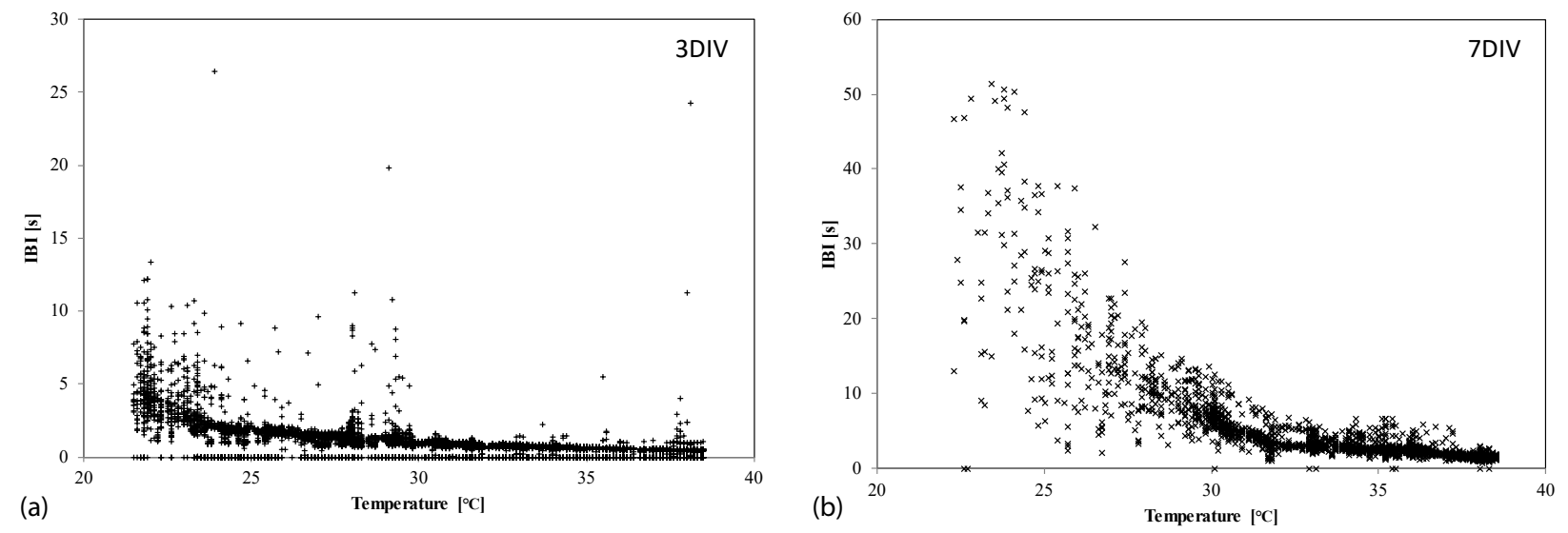

Figure 3. Temperature dependence of the inter-beat interval. The inter-beat interval (IBI) of synchronized beats in (a) an early stage culture (3 DIV) and (b) a late stage culture (7 DIV), at each temperature, and measured at one typical channel of an electrode in one sample. Note that the scale of the IBI axis in (a) is half that in (b)
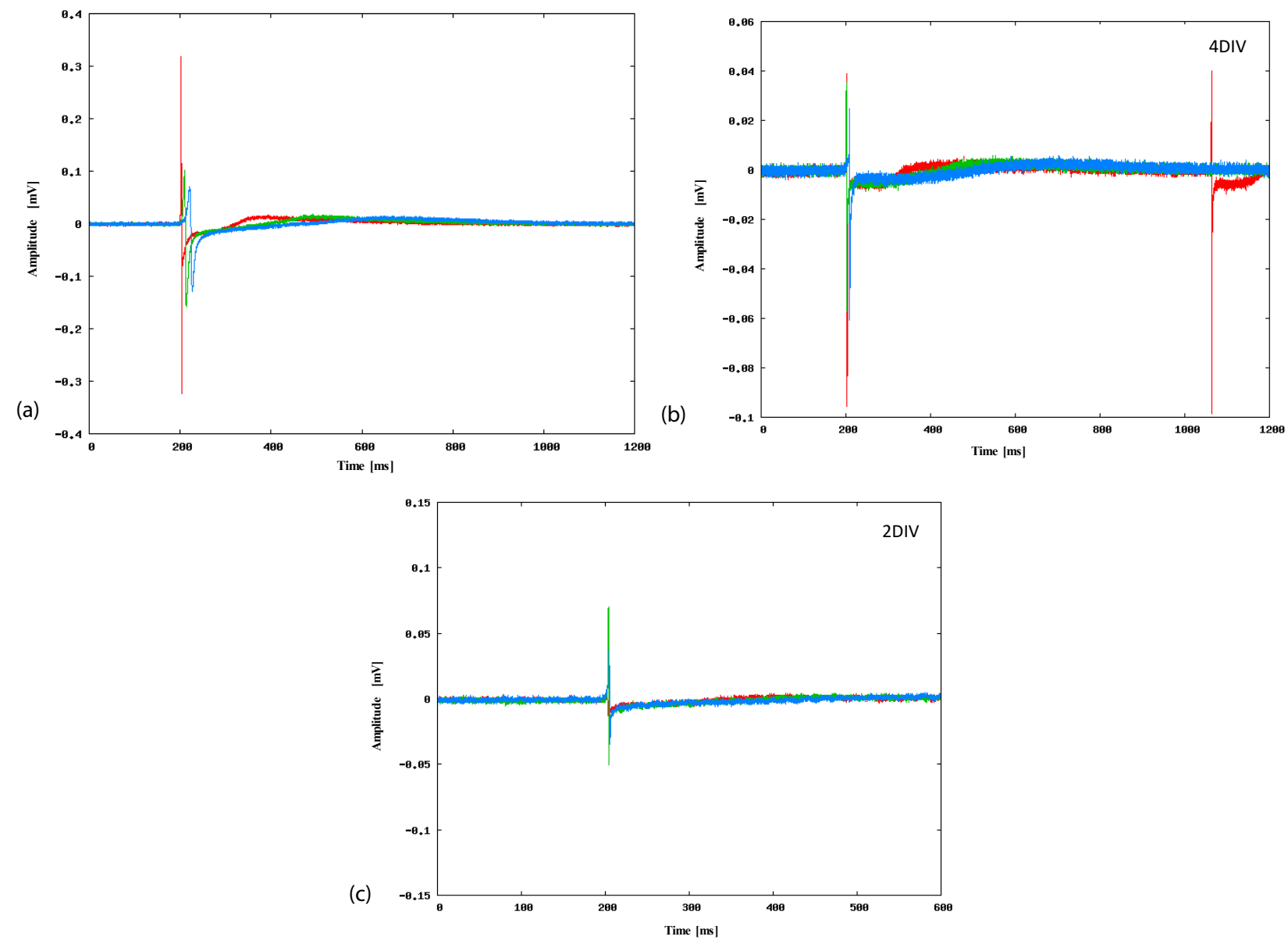

Figure 4. Typical field potential profiles measured using an MEA. Typical profiles of an electrode at three temperatures $\left(38^{\circ} \mathrm{C}\right.$ : red, $30^{\circ} \mathrm{C}$ : green, $22^{\circ} \mathrm{C}$ : blue) in (a) late culture stage $(7$ DIV), (b) middle culture stage (4 DIV), and (c) early culture stage (2 DIV). Note that the horizontal and perpendicular axes are different in order to show the profile in detail

beatings changed for several times during the measurements and the propagation velocity decreased. The propagation velocities were then estimated as $v_{L}=9.9 \pm 4.7 \mathrm{~cm} / \mathrm{s}$ at $30^{\circ} \mathrm{C}$ (Figure $5 \mathrm{~b}$ ) and $v_{L}=4.8 \pm 2.0 \mathrm{~cm} / \mathrm{s}$ at $22^{\circ} \mathrm{C}$ (Figure $5 \mathrm{c}$ ). The microscopic observations indicated that, in the late stage cultures, the synchronicity of the beating was maintained even at the lowest temperature, and that the number of electrodes that measured the field potential in the late stage culture was $96 \pm 4 \%$ of that at $38^{\circ} \mathrm{C}$ (Figure $\mathrm{S} 4$; Video in the Supporting Information).
In the early stage culture, fewer electrodes measured the field potential (approximately 50-35\%; Figure 6 upper column) even at the physiological temperature. This was mainly because the isolated cardiomyocytes were widely distributed on the MEA and some of them were not located on the electrode. The number of electrodes measuring the field potential fell further to $41 \pm 15 \%$ of that at $38^{\circ} \mathrm{C}$ at the lowest temperature. This number varied mainly because the intensity of the field potential was already small at $38^{\circ} \mathrm{C}$, and it could not be 

arrays

distinguished with the noise at some electrodes at $22^{\circ} \mathrm{C}$, although the microscopic observations confirmed that most of the cardiomyocytes kept beating (Figure S2; Video in the Supporting Information). The starting point of the synchronized beating was not always fixed, even under physiological conditions. Since the offset-time interval vs distance diagrams (Figure 6 lower column) were almost linear, we assume that each wave of synchronized beating was concentrically circular. Then we estimated the propagation velocities by defining the starting electrode in each beating profile. The propagation velocities were found to be lower than those obtained in the late stage cultures: $v_{E}=5.9 \pm 2.4 \mathrm{~cm} / \mathrm{s}$ at $38^{\circ} \mathrm{C}, 5.2 \pm 1.6 \mathrm{~cm} / \mathrm{s}$ at $30^{\circ} \mathrm{C}$, and $3.5 \pm 0.7 \mathrm{~cm} / \mathrm{s}$ at $22^{\circ} \mathrm{C}$ (Figures $6 a-$ $6 \mathrm{c})$, but their temperature dependence was smaller than that at the late stage of culture (Figure 7a). Statistical analysis indicates that there are slight differences on the temperature dependences of the propagation velocities between culture stages (except at $22^{\circ} \mathrm{C}$ ) and between three temperatures $(\mathrm{p}=0.02$, by the Tukey-Kramer test). (a) $38^{\circ} \mathrm{C}$
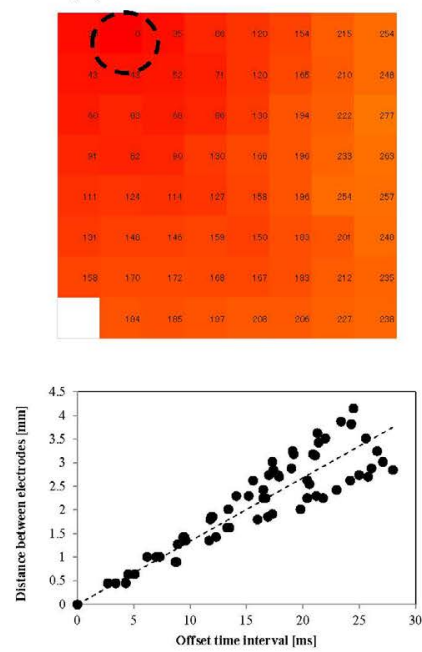

(b) $30^{\circ} \mathrm{C}$
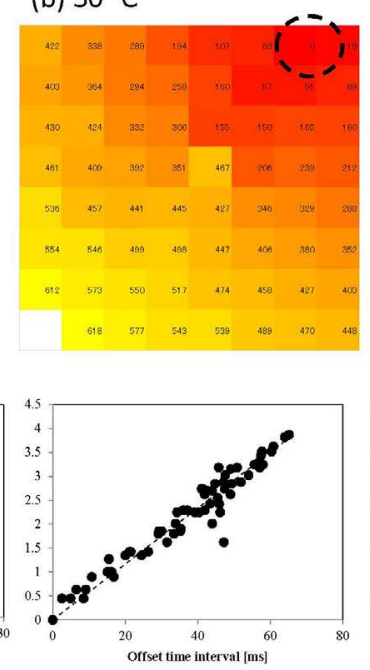

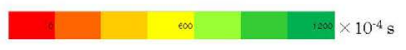

(c) $22^{\circ} \mathrm{C}$
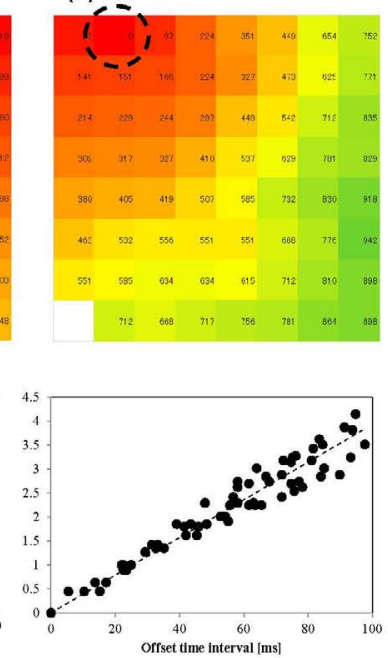

Figure 5. Typical propagation map of a synchronized beat of a late stage culture. Activation-time maps (upper) and electrode-distance vs. time-interval graphs (lower) on the late stage sample at (a) $38^{\circ} \mathrm{C}$, (b) $30^{\circ} \mathrm{C}$, and (c) $22^{\circ} \mathrm{C}$. The dashed circle in the activation-time map denotes the starting electrode of a beat. The offset time interval from the starting electrode is measured in milliseconds and is shown by different colors. The offset time interval is related to the distance (mm) in order to evaluate the average propagation velocity by the least-squares method

(a) $38^{\circ} \mathrm{C}$

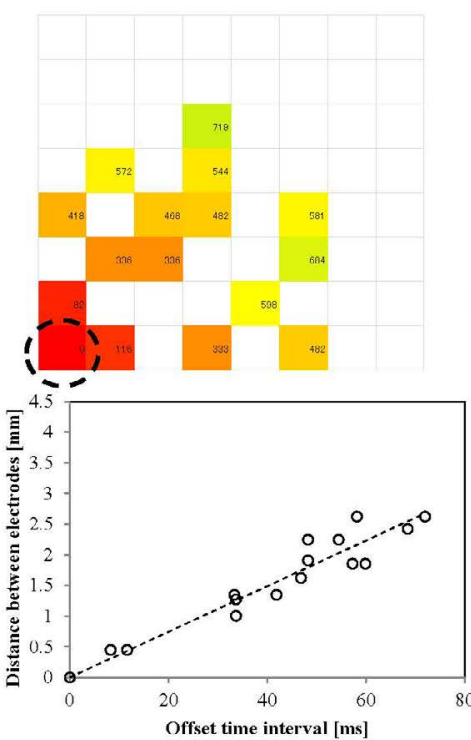

(b) $30^{\circ} \mathrm{C}$
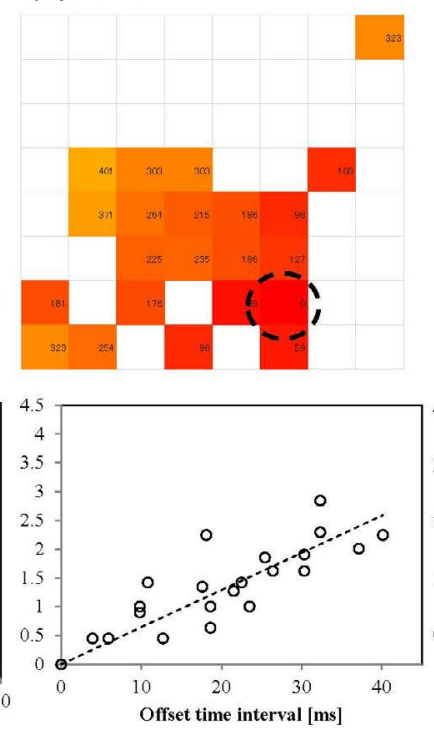

(c) $22^{\circ} \mathrm{C}$
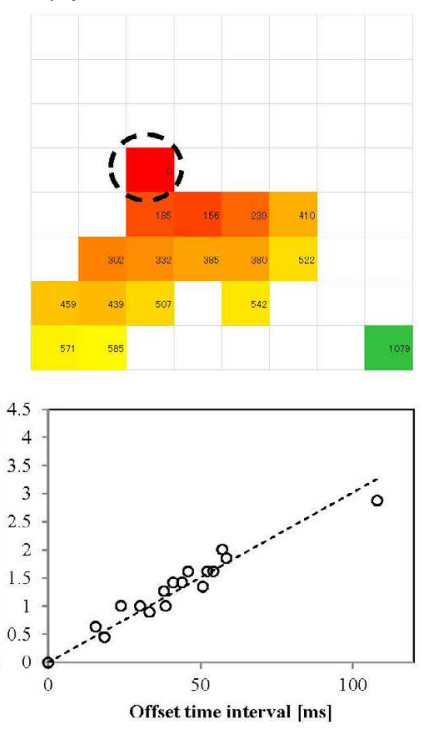

Figure 6. Typical propagation map of a synchronized beat of an early stage culture. Activation-time maps (upper) and electrode-distance vs. time-interval graphs (lower) on the early stage sample at (a) $38^{\circ} \mathrm{C}$, (b) $30^{\circ} \mathrm{C}$, and (c) $22^{\circ} \mathrm{C}$. The dashed circle in the activation-time map denotes the starting electrode of a beat. The offset time interval from the starting electrode is measured in milliseconds and is shown by different colors. The offset time interval is related to the distance (mm) in order to evaluate the average propagation velocity by the least-squares method 

arrays

We then analyzed the signal propagation characteristics by assuming the thermal-activation process:

$$
v_{i}=\mathrm{v}_{\mathrm{i}}^{0} \exp \left[-E_{i} / \mathrm{k} T\right],(1)
$$

where $_{\mathrm{i}}$ is either ${ }_{\mathrm{E}}$ (early stage) or ${ }_{\mathrm{L}}$ (late stage), $\mathrm{v}_{\mathrm{i}}^{0}$ is constant, $E$ is the activation energy, $\mathrm{k}$ is the Boltzmann constant, and $T$ is the absolute temperature. The Arrhenius plots of the propagation velocities (Figure $7 \mathrm{~b}$ ) indicate that the activation energy of the synchronized beating propagation $E_{L}$ was approximately $65 \mathrm{~kJ} / \mathrm{mol}$ (or the temperature coefficient $Q_{10}$ of 8.7$)$, and $E_{E}=25 \mathrm{~kJ} / \mathrm{mol}\left(Q_{10}\right.$ of 2.0$)$. The latter value is similar to that reported in a previous work $\left(Q_{10}\right.$ of 2.3) [27]. However, it is interesting that the temperature dependence of the signal propagation velocity varied with the culture period of the neonatal rat heart-cell network.

\section{Discussion}

\section{General maturation process of heart-cell cultured on MEAs}

Both the microscopic observations and the MEA measurements of the primary rat ventricle heart-cells that were cultured for about one week indicate that the cultured cardiomyocytes used for the present study had morphologies and beating properties similar to those observed in our previous studies [3,24]. We have also confirmed that the obtained results were reproducible within the experimental uncertainty.

The process of developing the heart-cell networks on MEAs can be summarized as follows. The dispersed cardiomyocytes (about 0.78 of seeded cells) start to beat spontaneously after they have adhered on a plate. When neighboring cells come into contact, their beating becomes synchronized by the formation of gap junctions between them (at the early stage of culture). In the middle stage, the cells on the MEA become confluent forming a cell sheet by mainly the multiplying of non-cardiomyocytes. Before the network being confluent, the heart cells form cell domains in which all cardiomyocytes beat synchronously. Even if the different cell domains beat at different frequencies, their beating becomes synchronized after the space between these domains is covered by the cells. When the cultured dish was almost confluent (about two-third of heart cells were non-cardiomyocytes), we considered this stage as the maturation of the heart-cell network. As the cultivation period progresses, the spontaneous synchronized beating becomes less and periodical at $38^{\circ} \mathrm{C}$. The previous studies $[28,29]$ showed this beating modulation to be resulted from the communications not only between cardiomyocytes but also between cardiomyocytes and non-cardiomyocytes through $\mathrm{Cx} 43$. Our results related to the average beating-rate change [24] and the increase of non-cardiomyocytes with the cultivation period may support the effect of non-cardiomyocytes on the signal propagation in the heart-cell network. Since the signal propagation rate was large at this stage, the synchronized beating of the heart-cell network was almost simultaneous within the observed area (about $4 \times 4 \mathrm{~mm}^{2}$; Figure 5a upper column and Figure S3 in the Supporting Information).

\section{Decrease of beating rate at lower temperatures}

We found that the beating rate of cardiomyocytes decreased as the temperature decreased to about $22^{\circ} \mathrm{C}$. This result qualitatively coincides with previous reports on cardiomyocytes [10,30]. Thus, we have confirmed that the beating activity of cardiomyocytes in the heart-cell network decreases as temperature decreases. In addition, we investigated the variation of temperature sensitivity of the beating rate with the duration of the culture period.

The field potential profiles showed that the action potential duration was elongated significantly at lower temperatures in the late stage culture (Figure $4 \mathrm{a}$ ). This is consistent with previous results [26,30]. The mechanism underlying this activity depression at lower temperatures is thought to be as follows: the ATP production rate decreases, whereas the demand on ATP increases, because the activation of ion channels and ion pumps in order to accelerate depolarization and repolarization. The decrease in the activity of ion channels and ion pumps is also caused by the decrease in the ion concentration gradient across the cell membrane, because the permeability of the membrane to ions increases at lower temperatures [26]. Thus, the temperature drop depresses the speed of ion exchange, which results in the accumulation of $\mathrm{Ca}^{2+}$ in the cardiomyocytes and the elongation of the contraction-relaxation cycle of sarcomeres [8]. As shown in Figure 4a, we demonstrated a significant delay in later positive peaks in the late stage cultures. However, these elongation processes do not appear clearly at the early culture stage (Figure 4c), and the slight delays in later positive peaks were observed in the middle stage samples (Figure $4 \mathrm{~b}$ ). Thus, we propose that the temperature sensitivity increased with the maturation of the heart-cell network, which was observed first in the delay in the $\mathrm{K}^{+}$current and
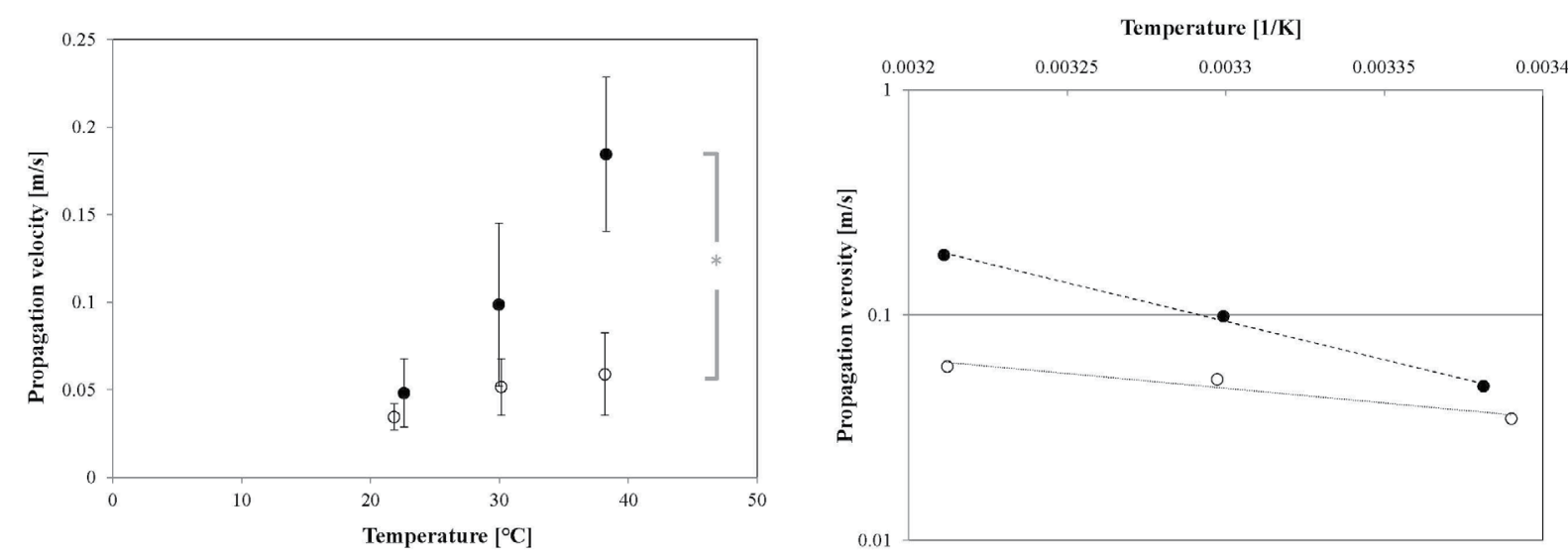

Figure 7. Temperature dependence of propagation velocity of a synchronized beat. (a) Temperature dependence of the average propagation velocity of the beating signal in the cardiomyocyte sheet at the early culture stage (open circles) and late culture stage (solid circles). Each error bar shows S.D. of propagation velocity at each temperature. Statistical analysis indicates that there is a slight difference between each culture stage $\left(*\right.$ : $p<0.05$, by a Tukey-Kramer test), except at $22^{\circ} \mathrm{C}$. (b) Arrhenius plot of the propagation velocities shown in (a). The dotted and dashed lines are the curves fitted with the least-squares method for the early stage $\left(R^{2}=0.935\right)$ and late stage $\left(R^{2}=0.997\right)$ cultures, respectively 

arrays

then in the delay and decrease in the $\mathrm{Na}^{+}$current. Therefore, immature cardiomyocytes may be insensitive to temperature changes even in non-hibernating mammals.

The mechanisms that protect cardiomyocytes at low temperatures in hibernating mammals have been studied previously. Kondo [8,31,32] showed that the cardiomyocytes of hibernating chipmunks were still active at low temperatures when the special $\mathrm{Ca}^{2+}$ current control system was expressed. Since this $\mathrm{Ca}^{2+}$ current control system is driven by low $\mathrm{K}^{+}$ channel activity [31], the low-temperature sensitivity of the $\mathrm{K}^{+}$current in the early stage cultures of rat cardiomyocytes would play a similar role in preventing $\mathrm{Ca}^{2+}$ accumulation in the cell. Thus, rat heart cells in the early culture stage would have a higher tolerance to cold conditions than those in later stages. Thus we consider that the injuries of heart cells would be negligible small during the sample transportation prior to the experiments.

Furthermore, the field potential profiles of cardiomyocytes must be essential for maintaining the constant frequency of the synchronized beating. These complex regulatory systems would be acquired during the maturation of the network. However, the temperature sensitivity of these networks would increase simultaneously.

\section{Mechanism underlying the decrease in the propagation velocity of a beating signal at lower temperatures}

We consider that the decrease in beating activity at lower temperatures was caused not only by the decrease in the synchronized beating rate but also by the reduced propagation velocity of the field potential signal within the heart-cell network. We have revealed that these characteristics differ depending on the duration of the culture period and depending on the maturity of the cell network. In order to consider the effects of low temperature on the propagation velocity of a field potential, we consider two processes to be factors: (1) the increase in the duration of an action potential (ion exchange duration) and/or a single stroke of beating (mechanical duration) of a cardiomyocyte within the network, and (2) the temporal delay in the intercellular communication in the network. Since the former process has already been discussed in the previous section, we only discuss the latter process here.

We have found that the temperature dependence of the propagation velocity of the field potential in a heart-cell network differed according to the culture stage. In early stage cultures, the average activation energy of the signal propagation was $E_{E}=25 \mathrm{~kJ} / \mathrm{mol}$. This value coincides with the activation energy of the channel conductance of the gap junction, $23.8 \mathrm{~kJ} / \mathrm{mol}$, which was obtained from ventricular myocytes of neonatal SD rats after 1-2 DIV [33]. Thus, we consider that the gapjunction channels formed between cardiomyocytes at the early stage (Figure 1b) behave like 'water-filled pores' [33]. Since the rate of noncardiomyocytes in the network was small in early stage, the effect of the non-cardiomyocytes on the signal propagation would be small.

However, the activation energy of the signal propagation in the late stage cultures is $E_{L}=65 \mathrm{~kJ} / \mathrm{mol}$. This is much greater than $E_{E}$, although it might include the elongation effect of the mechanical duration of the cardiomyocytes. Here we assume that the effect of the gap-junction channels on the signal propagation in the network develops during the maturation of the heart-cell network and contributes to the change of activation energy. This is based on the observation that the number concentration of $\mathrm{Cx} 43$ observed at the cell-to-cell boundaries increased with the culture duration (Figure 1). Although we could not define the interaction between cardiomyocytes and non-cardiomyocytes precisely, the modulation of the synchronized beating rate with the cultivation period suggests there are several communications between them through $\mathrm{Cx} 43[28,29]$. Although this heterocellular interaction would contribute to the change of the activation energy, it may be small compared to the interaction between cardiomyocytes since we observed the expression of $\mathrm{Cx} 43$ mostly at the boundaries between cardiomyocytes.

High activation energy on $E_{L}$ would suggest that the gap-junction channel involves some biochemical processes, such as 'channel gating' [33]. It is also notable that the late stage cultures tended to suffer from $\mathrm{Ca}^{2+}$ accumulation within the cardiomyocytes. The increase in the $\mathrm{Ca}^{2+}$ concentration in a cell accelerates the obstruction of gapjunction channels [34], and this decreases the propagation velocity of the intercellular action potential signal by increasing gap-junction conductance. Therefore, we consider that the delay in the propagation velocity of the intercellular field potential signal in late stage cultures is a result of the increase in the gap-junction conductance due to the functional change of these channels from 'water-filled pores' to 'gated channels' and to the obstruction of the ion-current by the accumulation of $\mathrm{Ca}^{2+}$ in the cell. This assumption would be checked by the $\mathrm{Ca}^{2+}$ concentration measurements in the future.

\section{Increase in IBI variability at low temperatures}

When the environmental temperature decreased, we found that the heart-cell networks in the early stage cultures elongated the IBI with maintaining the periodic synchronized beating. However, those in late stage cultures (or in a matured heart-cell network) could not maintain periodic beating at temperatures below $30^{\circ} \mathrm{C}$ (Figure 3).

Since we observed the synchronized beating without any stimulation in this experimental system, the constant and periodic synchronized beating was generated by a pacemaker myocyte in the network. As discussed previously, the IBI of the pacemaker myocyte increased as the temperature decreased. If the IBI exceeded the intrinsic beating interval of a non-pacemaker myocyte with a shorter interval, this myocyte act as an alternative pacemaker with a different beating rhythm.

The inhibition of intercellular communications through gapjunction channels would also make it difficult to regulate the periodic beating in the network. In this case, the IBI variability would increase at lower temperatures. For example, the IBI variability increased sharply upon exceeding $5 \mathrm{~s}$ at temperatures below $30^{\circ} \mathrm{C}$ in the late stage cultures (Figure $3 \mathrm{~b}$ ). At that moment, the starting point of the synchronized beating changed (Figure $5 \mathrm{~b}$ ). Since the new IBI was shorter than before, the decrease in the beating rate would be smaller as the temperature decreased. This would result in the existence of the inflexion point in the temperature dependence of the beating rate for the late stage cultures (Figure 2).

Conversely, the increase in IBI variability as the temperature dropped was relatively small in the early stage samples. Figure $3 \mathrm{a}$ shows that IBI peaked at approximately $5 \mathrm{~s}$ at the lowest temperature. Compared with the late stage cultures, the range of IBI variability of pacemaker myocytes would be smaller than that of non-pacemaker myocytes. When we consider that the starting point of the synchronized beating was not always fixed even under physiological conditions, the small variability of IBI at the early stage indicates that the IBI would be similar for most cardiomyocytes in the system. It would be because the weak intercellular connections do not regulate the periodic beating as strictly. 

arrays

The previous studies indicated that the loss of periodic beating at lower temperatures resulted in severe injury to cardiac cells [9], cardiac tissues [8,26], hearts [35], and adult rats [7]. Although we cannot yet explain in detail the reason why arrhythmia occurs at low temperature from our results, the increase in the IBI of synchronized beating and the obstruction of the signal propagation at the gap junctions would contribute to the arrhythmia seriously injuring the individual by reducing not only the contracting activity, due to the irregularity of the beating, but also the survival of heart cells themselves due to the accumulation of $\mathrm{Ca}^{2+}$. The present study indicates that the development of gap junctions in the middle to late stage cultures would make intercellular communication robust and smooth. However, the system would simultaneously become sensitive to, and subject to damage severely from the temperature drop. Conversely, immature cardiac networks would not suffer severe damage under hypothermic conditions, suggesting the possibility of primary cardiomyocyte-cell preservation at lower temperatures [24].

\section{Conclusions}

The synchronized beating properties of neonatal SD rat ventricle heart-cells cultured on MEAs were simultaneously examined using an optical microscope and with micro-electrode arrays at temperatures between $38^{\circ} \mathrm{C}$ and $22^{\circ} \mathrm{C}$. At the early culture stage (1-3 DIV), most of the cardiomyocytes were still isolated but were beating spontaneously at relatively high beating rates. Several cardiomyocytes became partly connected by forming gap junctions, and their beating became synchronized. The temperature dependence of the synchronized beating rate was almost linear and reversible within the range of the present experimental conditions. The temperature dependence of the propagation velocity of field potentials in the network indicated that the gap junctions acted as 'water-filled pores'.

However, after subsequent culture, the heart-cell networks developed to be confluent with cell-to-cell connecting gap-junctions increase, with the propagation velocity being large, and with periodically synchronized beating. This maturation of the heart-cell network modified its temperature dependence, as shown by the large increase in the IBI variability below $30^{\circ} \mathrm{C}$, as well as the large drop in the signal propagation velocity. The activity drop of each cardiomyocyte at low temperatures was caused by the ATP deficiency and the eventual accumulation of $\mathrm{Ca}^{2+}$. These processes would result in the delay of intercellular communications because the gap junctions would be acquired a gating function during the maturation of the network. Thus, the exposure of mature heart-cell networks to low temperatures resulted in more severe injuries to them than those of immature heartcell networks.

\section{Acknowledgments}

We thank K. Miyamura for the time-lapse observations of heart cells, and S. Taniwaki and D. Ito for their technical supports of experiments. Primary rat ventricle heart-cells and their culture medium were supplied by T. Taira, K. Shimizu and M. Sakai (Cosmo Bio Co., Ltd.). We also acknowledge the fruitful discussion with M. Nagayama (Hokkaido University of Education, Asahikawa Campus). The authors have no conflicting financial interests.

\section{Funding}

This work was supported by a Grant-in-Aid for Scientific Research from the Japan Society for the Promotion of Science (Grant No. 23350001 and 17K1883407) from the Japan Science and Technology Agency.

\section{Author contributions}

Conceiving and designing the experiments: TU, KG. Performing the experiments: RK. Analyzing the data: TU, RK. Writing the paper: TU, RK, KG.

\section{References}

1. Chalopcikova S, Psotova J, Miketova P (2001) Neonatal rat cardiomyocytes -A model for the study of morphological, biochemical and electrophysiological characteristics of the heart. Biomed Papers 145: 49-55.

2. Haraguchi Y, Shimizu T, Yamato M, Kikuchi A, Okano T (2006) Electrical coupling of cardiomyocyte sheets occurs rapidly via functional gap junction formation. Biomaterials 27: 4765-4774.

3. Miyamura K, Nagayama M, Gohara K, Taira T, Shimizu K, et al. (2010) Evaluation of viability of cryopreserved rat cardiac myocytes and effects of dimethyl sulfoxide concentration on cryopreservation. Cryobio Cryotech 56: 111-117.

4. Grice WN, Konishi T, Apstein CS (1987) Resistance of neonatal myocardium to injury during normothermic and hypothermic ischemic arrest and reperfusion. Circulation 76 : $\mathrm{V}-150-\mathrm{V}-155$

5. Jarmakani JM, Nakazawa N, Nagatomo T, Langer GA (1978) Effect of hypoxia on mechanical function in the neonatal mammalian heart. Am J Physiol 235: H469-H474

6. Bove EL, Gallagher KP, Drake DH, Lynch MJ, Fox M, Forder J, Boling SF, Shlafer M (1988) The effect of hypothermic ischemia on recovery of left ventricular function and preload reserve in the neonatal heart. $J$ Thorac Cardiovasc Surg 95: 814-818.

7. Matthew CB, Bastille AM, Gonzalez RR, Sils IV (2002) Heart rate variability and electrocardiogram waveform as predictors of morbidity during hypothermia and rewarming in rats. Can J Physiol Pharm 80: 925-933.

8. Kondo N (1997) Physiological and biochemical studies on hibernation contro mechanism in mammalian hibernators. In Sleep and Sleep Disorders: From Molecule to Behavior. Osaka: Takeda Sci Found, pp, 129-143.

9. Orita H, Fukasawa M, Hirooka S, Fukui K, Kohi M, Washio M (1993) A cardiac myocyte culture system as an in vitro experimental model for the evaluation of hypothermic preservation. Surg Today 23: 439-443.

10. Takayama S, Sakurai T, Hanaya T, Arai S, Ohta T, Fukuda S (2006) Persistent beating of cardiomyocytes from Syrian Hamster (Mesocricetus auratus) at low temperature. ITE Lett Batter New Technol Med 7: 302-307.

11. Harary I, Farley B (1963) In vitro studies on single beating rat heart cells. I. Growth and organization. Exp Cell Res 29: 451-465. [Crossref]

12. Orita H, Fukasawa M, Hirooka S, Uchino H, Fukui K, et al. (1993) Modulation of cardiac myocyte beating rate and hypertrophy by cardiac fibroblasts isolated from neonatal rat ventricle. Jpn Circ J 57: 912-920. [Crossref]

13. Oyamada M, Kimura H, Oyamada Y, Miyamoto A, Ohshika H, Mori M (1994) The expression, phosphorylation, and localization of connexin 43 and gap-junctional intercellular communication during the establishment of a synchronized contraction of cultured neonatal rat cardiac myocytes. Exp Cell Res 212: 351-358.

14. Yamauchi Y, Harada A, Kawahara K (2002) Changes in the fluctuation of inter-beat intervals in spontaneously beating cultured cardiac myocytes: experimental and modeling studies. Biol Cybern 86: 147-154.

15. Yoneyama M, Kawahara K (2004) Coupled oscillator systems of cultured cardiac myocytes: Fluctuation and scaling properties. Phys Rev E 70: 021904-1-9.

16. Kojima K, Kaneko T, Yasuda K (2005) Stability of beating frequency in cardiac myocytes by their community effect measured by agarose microchamber chip. $J$ Nanobiotech 3: 4-1-6

17. Kojima K, Kaneko T, Yasuda K (2006) Role of the community effect of cardiomyocyte in the entrainment and reestablishment of stable beating rhythms. Biochem Biophys Res Commun 351: 209-215. [Crossref]

18. Hachiro T, Kawahara K, Sato R, Yamauchi Y, Matsuyama D (2007) Changes in the fluctuation of the contraction rhythm of spontaneously beating cardiac myocytes in cultures with and without cardiac fibroblasts. Biosystems 90: 707-715.

19. Thomas SP, Bircher-Lehmann L, Thomas SA, Zhuang J, Saffitz JE, Kleber AG (2000) Synthetic strands of neonatal mouse cardiac myocytes, structural and electrophysiological properties. Circ Res 87: 467-473.

20. Meiry G, Reisner Y, Feld Y, Goldberg S, Rosen M, Ziv N, Binah O (2001) Evolution of action potential propagation and repolarization in cultured neonatal rat ventricular myocytes. J Cardiovasc Electrophysiol 12: 1269-1277. 

arrays

21. Reppel M, Pillekamp F, Lu ZJ, Halbach M, Brockmeier K, et al. (2004) Microelectrode arrays: a new tool to measure embryonic heart activity. J Electrocardiol 37 Suppl: 104 109. [Crossref]

22. Meyer T, Boven KH, Günther E, Fejtl M (2004) Micro-electrode arrays in cardiac safety pharmacology: a novel tool to study QT interval prolongation. Drug Saf 27: 763772. [Crossref]

23. Halbach MD, Egert U, Hescheler J, Banach K (2003) Estimation of action potential changes from field potential recordings in multicellular mouse cardiac myocyte cultures. Cell Physiol Biochem 13: 271-284. [Crossref]

24. Uchida T, Nagayama M, Taira T, Shimizu K, Sakai M, Gohara K (2011) Optimal temperature range for low-temperature preservation of dissociated neonatal rat cardiomyocytes. Cryobiology 63: 279-284.

25. Nagayama M, Uchida T, Gohara K (2007) Temporal and spatial variations of lipid droplets during adipocyte division and differentiation. J Lipid Res 48: 9-18.

26. Wang SQ, Cao HM, Zhou ZQ (1997) Temperature dependence of the myocardial excitability of ground squirrel and rat. $J$ Therm Biol 22: 195-199.

27. Shah U, Bien H, Entcheva E (2006) Cardiac arrhythmogenesis and temperature. Conf Proc IEEE Eng Med Biol Soc 1: 841-844. [Crossref]

28. Miragoli M, Salvarani N, Rohr S (2007) Myofibroblasts induce ectopic activity in cardiac tissue. Circ Res 101: 755-758. [Crossref]
29. Kizana E, Ginn SL, Smyth CM, Boyd A, Thomas SP, et al. (2006) Fibroblasts modulate cardiomyocyte excitability: implications for cardiac gene therapy. Gene Therapy 13: $1611-1615$

30. Chen YJ, Chen YC, Chan P, Lin CI, Chen SA (2003) Temperature regulates the arrhythmogenic activity of pulmonary vein cardiomyocytes. J Biomed Sci 10: 535-543. [Crossref]

31. Kondo N (1986) Excitation-contraction coupling in myocardium of nonhibernating and hibernating chipmunks: effects of isoprenaline, a high calcium medium, and ryanodine. Circ Res 59: 221-228.

32. Kondo N (1988) Comparison between effects of caffeine and ryanodine on electromechanical coupling in myocardium of hibernating chipmunks: role of internal Ca stores. Br J Phamacol 95: 1287-1291.

33. Bukauskas FF, Weingart R (1993) Temperature dependence of gap junction properties in neonatal rat heart cells. Pflugers Arch 423: 133-139. [Crossref]

34. Loewenstein WR (1981) Junctional intercellular communication: the cell-to-cell membrane channel. Physiol Rev 61: 829-913. [Crossref]

35. Langer SF, Lambertz M, Langhorst P, Schmidt HD (1999) Interbeat interval variability in isolated working rat hearts at various dynamic conditions and temperatures. Res Exp Med (Berl) 199: 1-19. [Crossref]

Copyright: (C2018 Uchida T. This is an open-access article distributed under the terms of the Creative Commons Attribution License, which permits unrestricted use, distribution, and reproduction in any medium, provided the original author and source are credited. 